\title{
High dietary cholesterol and ovariectomy in rats repress gene expression of key markers of VLDL and bile acid metabolism in liver
}

\author{
Zahra Farahnak, Isabelle Côté, Emilienne T. Ngo Sock and Jean-Marc Lavoie*
}

\begin{abstract}
Background: The purpose of the study was to evaluate the effects of high dietary cholesterol in ovariectomized $(\mathrm{Ovx})$ rats on several key markers of hepatic cholesterol and bile acid metabolism.

Method: Ovx and sham operated (Sham) rats were given either a standard diet (SD), a SD diet supplemented with $0.25 \%$ cholesterol (SD + Chol), or a high fat diet supplemented with $0.25 \%$ cholesterol (HF + Chol) for 5 weeks.

Results: Ovx was associated with higher $(P<0.05)$ liver total cholesterol $(T C)$ under the SD and the SD + Chol diet, while liver triglyceride (TG) content was higher in Ovx than in Sham rats in all 3 diet conditions. Surprisingly, the SD + Chol diet was associated with lower $(P<0.001)$ plasma TC and TG levels in Ovx than in Sham rats, suggesting a decrease in VLDL secretion. Accordingly, several transcripts of key markers of VLDL synthesis including microsomal TG transfer protein $(M t t p)$ and Apob-100 were decreased $(P<0.05)$ in Ovx compared to Sham rats under the three dietary conditions and even more so for Mttp and Apob-100 when rats were fed the SD + Chol diet. Transcripts of bile acid transporters including bile salt export pump (Bsep) and $\mathrm{Na}+$-taurocholate cotransporting polypeptide (Ntcp) were decreased by the addition of cholesterol to the SD diet in both Ovx and Sham rats.
\end{abstract}

Conclusion: These results indicate that a high cholesterol feeding and ovariectomy combine to reduce the gene expression of key markers of VLDL synthesis suggesting a reduction in excretion of cholesterol from the liver.

Keywords: Liver cholesterol, Cholesterol diet, VLDL synthesis, LDL receptors

\section{Introduction}

There has been accumulating evidence in recent years that estrogens deficient state in ovariectomized (Ovx) animals and in postmenopausal women results in substantial liver triglyceride (TG) accumulation [1-4]. On the other hand, evidence of disturbances of cholesterol metabolism in link with estrogens deficiency is limited to observations of higher plasma levels of total cholesterol found in human as well as in animal models [5-8]. Liver cholesterol metabolism in Ovx animals has received little attention and shows some controversies. For instance, liver total cholesterol (TC) level was reported not to be affected by Ovx in previous studies $[9,10]$ although we recently found an increase in rats ovariectomized for 8 wks [11]. There is, therefore, a need for

\footnotetext{
* Correspondence: jean-marc.lavoie@umontreal.ca Département de Kinésiologie, Université de Montréal, C.P. 6128, Succ. centre-ville, Montréal, Québec H3C 3 J7, Canada
}

more physiological and molecular information to better understand how liver, as a master regulator of cholesterol metabolism, is affected by estrogens withdrawal.

Nutritional approaches have been used frequently as a tool to investigate the role of the liver in regulating TG and cholesterol metabolism [12, 13]. In Ovx animals, our group recently observed a large cholesterol accumulation in liver of rats fed a high fat (HF) diet, suggesting a vulnerability to cholesterol accumulation of Ovx animals when fed a HF diet [14]. The vulnerability of Ovx animals to dietary cholesterol has also been recently enlighten by the demonstration that gene expression of several molecular markers of VLDL assembly were reduced following high fat/high cholesterol diets [15]. However, dietary fat and dietary cholesterol have been reported to result in a positive synergistic interaction on the development of for instance hypercholesterolemia [13]. We, therefore, postulated that a better understanding of how 
Ovx animals regulate hepatic cholesterol metabolism would be obtained if the animals were fed a high cholesterol diet without the confounding effect of dietary fat.

In an attempt to shed some light on how liver of Ovx animals respond to high dietary cholesterol, we targeted key molecular markers of pathways involved in cholesterol/bile acids metabolism/transport that have recently been found to be affected by estrogens deficiency [15]. We first looked at molecular markers of VLDL assembly, including microsomal TG transfer protein $(M t t p)$, a rate limiting molecule in VLDL assembly and secretion, Apob100 an essential structural protein that translocates into the luminal side of the endoplasmic reticulum, diacylglycerol acyltransferase 2 (Dgat2) involved in converting fatty acids into triglyceride (TG), acyl-Coa: cholesterol acyl transferase (Acat2) that converts free cholesterol into cholesterol ester, cell death-inducing like-effector type B (Cideb) a protein involved in lipidation of particles, and small GTP-binding protein a (Sar1a) a protein that facilitates the movements of VLDL particles toward the Golgi apparatus.

Furthermore, we investigated the gene expression of molecular markers of bile acids metabolism/transport, a pathway that is tightly associated with elimination of cholesterol from the liver. These included ATP-cassette binding protein G5 and G8 (Abcg5/Abcg8) that export cholesterol from hepatocytes to the bile duct, bile salt export pump (Bsep) and multidrug resistance-associated transporter 2 (Mdr2) which stimulate bile acid and phospholipid transport from hepatocytes to bile canaliculi, $\mathrm{Na}+$-taurocholate cotransporting polypeptide $(N t c p)$ involved in bile acid uptake in the basolateral membrane of the hepatocytes, farnasoid X receptor $(F x r)$ a nuclear receptor involved in regulation of hepatic bile acid biosynthesis, and cytochrome P450 7A1 (Cyp7a1) the main enzyme that catalyses the conversion of cholesterol into bile acids. Finally, we complemented our approach by investigating the response of hepatic LDL-receptor $(L d l-r)$, a major determinant of removal of LDLcholesterol particles from the circulation, LDL receptorrelated protein-1 (Lrp-1) involved in the removal of plasma remnant lipoproteins $[16,17]$, and sterol regulatory element-binding protein-2 (Srebp2) a transcription factor involved in the regulation of cholesterol.

The aim of the present study was to determine the effects of high dietary cholesterol on hepatic key markers of VLDL and cholesterol/bile acid metabolism in Ovx rats.

\section{Methods}

\section{Animal care}

Female Sprague-Dawley rats ( $n$ 48; Charles River, St Constant, PQ, Canada) weighing 180-200 g upon arrival were housed individually. Food and water were supplied ad libitum. Their environment was controlled in terms of light (12 h light-dark cycle starting at 06:00 AM), humidity and room temperature $\left(20-23{ }^{\circ} \mathrm{C}\right)$. Body weight and food intake were monitored two times per week. All experimental procedures were conducted according to the protocols approved by the directives of the Canadian Council on Animal Care after institutional approval.

\section{Diets and surgery}

Rats were first acclimated to their environment for a period of one week while fed a chow diet (12.5\% lipid, $63.2 \% \mathrm{CHO}$ and $24.3 \%$ protein; kJ from Agribrands Canada, Woodstock, Ontario, Canada). Thereafter, rats underwent either a bilateral ovariectomy (Ovx, $n$ 24) or a bilateral sham-operation (Sham, $n$ 24) according to the technique described by Robertson et al. under isoflurane anaesthesia [18]. After surgery, animals were injected with antibiotics (Tribrissen $24 \% ; 0.125 \mathrm{cc} / \mathrm{kg}$, subcutaneously) and analgesic (Carprofen; $4.4 \mathrm{mg} / \mathrm{kg}$, subcutaneously) for 3 days. Thereafter, the Ovx and Sham rats were assigned to one of three following diets: standard (SD), SD supplemented with $0.25 \%$ cholesterol (SD + Chol), or high fat supplemented with $0.25 \%$ cholesterol $(\mathrm{HF}+\mathrm{Chol})$ for 5 weeks (Table 1). The $0.25 \%$ cholesterol dose was chosen to increase the dietary cholesterol without the atherogenic effects of higher doses used in several other studies $[12,13]$.

\section{Blood and tissue sampling}

Rats were fasted overnight and sacrificed between 09:00 and 12:00 AM. Immediately after complete anaesthesia with isoflurane, the abdominal cavity was opened following the median line of the abdomen and approximately $4 \mathrm{ml}$ of blood was collected from the abdominal vena cava $(<45$ s) into syringes pre-treated with ethylenediaminetetraacetic acid (15\%; EDTA). Blood was centrifuged (3000 rpm; $4{ }^{\circ} \mathrm{C} ; 10 \mathrm{~min}$; Beckman GPR Centrifuge) and the plasma kept for further analyses. Immediately after blood collection, the liver median lobe was removed and freeze-clamped. This sample was used for triacylglycerol (TG), cholesterol, and mRNA determinations. Several organs and tissues were removed and weighed (Mettler $\mathrm{AE}$ 100) in the following order: uterus, mesenteric, urogenital, and retroperitoneal fat deposits. The mesenteric fat pad consisted of adipose tissue surrounding the gastrointestinal tract from the gastroesophageal sphincter to the end of the rectum. The urogenital fat pad included adipose tissue surrounding the kidneys, uterus and bladder as well as ovaries, oviducts and uterus. The retroperitoneal fat pad was taken as that distinct deposit behind each kidney along the lumbar muscles. All tissue samples were frozen in liquid nitrogen immediately after being weighed (Mettler AE-100). All tissue samples were stored along 
Table 1 Diet description

\begin{tabular}{|c|c|c|c|}
\hline & $\begin{array}{l}\text { Standard diet } \\
\text { (SD) (D12450J) }\end{array}$ & $\begin{array}{l}\text { SD + Chol (0.25 \%) } \\
\text { (D13020701) }\end{array}$ & $\begin{array}{l}\text { High Fat + Chol } \\
(0.25 \%)(\mathrm{D} 13020703)\end{array}$ \\
\hline \multicolumn{4}{|l|}{ (\%) } \\
\hline Protein & 19.2 & 19.2 & 22.8 \\
\hline Carbohydrate & 67.3 & 67.3 & 45.7 \\
\hline Fat & 4.3 & 4.3 & 20.2 \\
\hline \multicolumn{4}{|l|}{ (g) } \\
\hline Casein & 200 & 200 & 200 \\
\hline L-Cysine & 3 & 3 & 3 \\
\hline Corn Starch & 506.2 & 506.2 & 202.5 \\
\hline Maltodextrin 10 & 125 & 125 & 125 \\
\hline Sucrose & 68.8 & 68.8 & 68.8 \\
\hline $\begin{array}{l}\text { Cellulose, } \\
\text { BW200 }\end{array}$ & 50 & 505 & 50 \\
\hline Soybean oil & 25 & 25 & 25 \\
\hline Lard & 20 & 20 & 155 \\
\hline $\begin{array}{l}\text { Mineral Mix } \\
\text { S10026 }\end{array}$ & 10 & 10 & 10 \\
\hline $\begin{array}{l}\text { DiCalcium } \\
\text { Phosphate }\end{array}$ & 13 & 13 & 13 \\
\hline $\begin{array}{l}\text { Calcium } \\
\text { carbonate }\end{array}$ & 5.5 & 5.5 & 5.5 \\
\hline $\begin{array}{l}\text { Potassium } \\
\text { citrate, } 1 \mathrm{H} 2 \mathrm{O}\end{array}$ & 16.5 & 16.5 & 16.5 \\
\hline $\begin{array}{l}\text { Vitamin mix } \\
\text { V10001 }\end{array}$ & 10 & 10 & 10 \\
\hline $\begin{array}{l}\text { Choline } \\
\text { bitartrate }\end{array}$ & 2 & 2 & 2 \\
\hline Cholesterol & 0.0 & 2.63 & 2.63 \\
\hline $\mathrm{Kcal} / \mathrm{g}$ & 3.85 & 3.84 & 4.56 \\
\hline
\end{tabular}

Formulateds by: Research Diets, Inc. (20 Jules Lane, New Brunswick, NJ 08901 USA)

with plasma samples at $-80{ }^{\circ} \mathrm{C}$ until analyses were performed.

\section{Biochemical analyses}

Commercial kits from Sigma (Sigma; St-Louis, Missouri, USA) were used to determine plasma and liver TG by colorimetric method. Liver TG concentrations were estimated from glycerol released after $\mathrm{KOH}$ hydrolysis. Total liver cholesterol concentrations were determined with some adaptations of the procedure described by Folch et al. [19]. Briefly, $0.1 \mathrm{~g}$ of liver was homogenized with chloroform-methanol mixture $(2: 1, \mathrm{v} / \mathrm{v})$. The chloroform layer was collected and evaporated overnight. After adding $10 \%$ Triton X-100 in isopropanol, the sample was assayed for total cholesterol using commercial kits according to the manufacturer's instructions (Wako Diagnostics and Chemicals USA, Richmond, VA, USA). Plasma total cholesterol was determined using the same kits supplied by Wako.

\section{Molecular analyses}

Total RNA was extracted from frozen liver with the use of RNA extraction Mini kit (Invitrogen) according to the manufacturer's protocol. Then RNA was treated with DNase (Invitrogen) in order to avoid genomic contamination. Total RNA $(2 \mu \mathrm{g})$ was reverse-transcribed into complementary DNA using high capacity complementary DNA reverse transcription kits (Applied Biosystems). RT samples were stored at $-20{ }^{\circ} \mathrm{C}$. Gene expression for $\beta$ actin was determined using a pre-validated Taqman Gene Expression Assay (Applied Biosystems, Rn01462661, Foster City, CA). Gene expression level for target genes was determined using assays designed with the Universal Probe Library from Roche. The primer sets and UPL probe numbers are presented in Table 2. To validate the efficiency of the qPCR assays, we used a mix of the samples tested in the study.

The ABI PRISM ${ }^{\circ} 7900 \mathrm{HT}$ (Applied Biosystems) was used to detect the amplification level and was programmed with an initial step of $3 \mathrm{~min}$ at $95{ }^{\circ} \mathrm{C}$, followed by 40 cycles for $5 \mathrm{~s}$ at $95{ }^{\circ} \mathrm{C}$ and $30 \mathrm{~s}$ at $60{ }^{\circ} \mathrm{C}$. All reactions were run in triplicate and the average values of threshold cycle $\left(C_{\mathrm{T}}\right)$ were used for quantification. $\beta$-actin was used as endogenous control. The relative quantification of target genes was determined using the $\Delta \Delta C_{\mathrm{T}}$ method. Briefly, the $C_{\mathrm{T}}$ values of target genes were normalized to an endogenous control gene

Table 2 Oligonucleotide primers used for quantitative real-time polymerase chain reaction

\begin{tabular}{lll}
\hline Gene & Oligo FWD & Oligo REV \\
\hline MDR2 & ggcattctccatcatcctgt & cacttctgttgctttactgtgtca \\
BSEP & cggtggctgagagatcaaat & tgcgatagtggtggagaaca \\
ABCG5 & cggagagttggtgttctgtg & caccgatgtcaagtccatgt \\
ABCG8 & cagatgctggctatcataggg & ctgatttcatcttgccacca \\
ACAT-2 & cctcacagatgcgtttcaca & ctctgctcacttgccattttt \\
Apob & gatggagatgggagatgaggt & gggctcctcatcaacaagag \\
Cideb & gctccaatggcctgctaag & ttatgatcacagacacggaagg \\
Cyp7a1 & ggagcttatttcaaatgatcagg & cactctgtaaagctccactcactt \\
DGAT-2 & aggatctgccctgtcacg & gtcttggagggccgagag \\
HMG-CoAr & caaccttctacctcagcaagc & acagtgccacacacaattcg \\
LDLr & tgctactggccaaggacat & ctgggtggtcggtacagtg \\
LRP-1 & aatcgagggcaagatgacac & ccagtctgtccagtacatccac \\
Mttp & gcgagtctaaaacccgagtg & cactgtgatgtcgctggttatt \\
FXR & ccacgaccaagctatgcag & tctctgtttgctgtatgagtcca \\
Sar1a & gggcaaaccacaggaaag & cactgcacatgaacacttcca \\
SREBP-2 & gtgcagacagtcgctacacc & aatctgaggctgaaccagga \\
NTCP & aaaatcaagcctccaaaggac & ttgtgggtaccttttccaga \\
ActB & cccgcgagtacaaccttct & cgtcatccatggcgaact \\
GAPDH & ccctcaagattgtcagcaatg & agttgtcatggatgaccttgg \\
\hline
\end{tabular}


( $\beta$-actin $)\left(\Delta C_{\mathrm{T}}=C_{\mathrm{T} \text { target }}-C_{\mathrm{T}} \beta\right.$-actin $)$ and compared with a calibrator: $\left(\Delta \Delta C_{\mathrm{T}}=\Delta C_{\mathrm{T} \text { Sample }}-\Delta C_{\mathrm{T}}\right.$ Calibrator $)$. Relative expression (RQ) was calculated using the Sequence Detection System (SDS) 2.2.2 software (Applied Biosystems) and the formula is $\mathrm{RQ}=2_{\mathrm{T}}^{-\Delta \Delta C}$.

\section{Statistical analysis}

All data are presented as mean \pm SE. Statistical significance $(P<0.05)$ was determined using a 2 -way ANOVA for non-repeated measures with ovariectomy and diets as main factors. Fisher LSD post hoc test was used in the event of a significant interaction effect. For a significant diet effect without interaction, Fisher LSD from a oneway ANOVA was used.

\section{Results}

There was no difference in initial body weights for the 6 groups (means \pm SE: $190.6 \pm 1.9$ to $193.3 \pm 1.7$ ). Body weight, intra-abdominal fat pad weight and food intake measured at the end of the experiment were all significantly $(P<0.01)$ higher in Ovx than in Sham animals in the three dietary groups (Table 3 ). The addition of Chol to the SD diet did not result in any changes in both Ovx and Sham rats in body weight, intra-abdominal fat pad weight, and food intake when compared to animals fed the SD diet. However, food intake was significantly $(P<0.05)$ higher in rats fed the $\mathrm{HF}+\mathrm{Chol}$ diet as compared to the two other diets in both Sham and Ovx animals. Body weight and intra-abdominal fat pad weight also showed a strong tendency to be higher in rats fed the $\mathrm{HF}+\mathrm{Chol}$ diet with respect to the Sham and Ovx conditions $(P=0.06$ and $P=0.08$, respectively). Uterus weight was lower $(P<0.001)$ in Ovx than in Sham rats throughout the dietary conditions confirming total ovariectomy (Table 3).

\section{Plasma and liver lipid profile}

Liver TC levels in Sham rats were progressively higher $(P<0.01)$ following the $\mathrm{SD}, \mathrm{SD}+\mathrm{Chol}$ and $\mathrm{HF}+\mathrm{Chol}$ diets order (Fig. 1(a)). This was not observed, however, in Ovx animals. Liver TC levels were on the whole higher $(P<0.001)$ in Ovx than in Sham rats but not under the $\mathrm{HF}+\mathrm{Chol}$ diet (Fig. 1(a)). Liver TG levels were higher $(P<0.001)$ in Ovx than in Sham rats in all three dietary conditions (Fig. 1(b)). Liver TG levels were not affected by the SD + Chol diet while it was higher $(P<0.01)$ in the $\mathrm{HF}+\mathrm{Chol}$ diet compared to the SD diet in both Ovx and Sham animals. A completely different picture was observed for the TC and TG concentrations measured in plasma. Plasma TC levels were higher $(P<$ 0.05) in Ovx than in Sham rats under the SD and HF + Chol diets but largely lower $(P<0.001)$ in Ovx than in Sham animals under the $\mathrm{SD}+\mathrm{Chol}$ diet (Fig. 1(c)). On the whole, plasma TC levels in Ovx rats were lower $(P<0.05)$ under the two Chol diets as compared to the SD diet. On the opposite of liver TG, plasma TG levels were lower $(P<0.05)$ in Ovx than in Sham rats under the SD and SD + Chol diets (Fig. 1(d)). Plasma TG values were also lower $(P<0.001)$ in Sham rats fed the $\mathrm{HF}+$ Chol diet as compared to Sham rats in the 2 other diets. On the whole, TC and TG levels under the SD+Chol feeding were higher in liver of Ovx vs Sham animals while the opposite was found in plasma.

\section{Hepatic gene expression}

Gene expressions of key molecules involved in VLDL synthesis are presented in Fig. 2. On the whole, mRNA levels of 5 out of the 6 genes involved in VLDL synthesis (including Sarla and Cideb and with the exception of Acat2) were lower $(P<0.05)$ in Ovx than in Sham rats in all dietary conditions (only under the SD + Chol diet for Dgat2). The lowest $(P<0.01)$ values for Mttp, Dgat2, and Apob-100 transcripts were found in Ovx rats under the SD + Chol (as compared to the SD diet), suggesting that Ovx and the SD + Chol diet combine to decrease VLDL synthesis. Interestingly, we found that the decreased responses in gene expression of Mttp, Dgat2, Acat2, and Apob-100 under the SD + Chol diet were statistically attenuated (as compared to the SD diet) when rats were fed the HF + Chol diet (Fig. 2).

To complete the information we investigated genes involved in hepatic cholesterol and biliary acids transport (Fig. 3). With the exception of $M d r 2$ transcripts, we

Table 3 Anthropometric parameters and food intake

\begin{tabular}{|c|c|c|c|c|c|c|}
\hline \multirow[t]{2}{*}{ Variables } & \multicolumn{2}{|l|}{$\underline{S D}$} & \multicolumn{2}{|c|}{ SD + Chol (0.25 \%) } & \multicolumn{2}{|c|}{$\mathrm{HF}+\mathrm{Chol}(0.25 \%)$} \\
\hline & Sham & Ovx & Sham & Ovx & Sham & Ovx \\
\hline Final body weight (g) & $353.9 \pm 11.3$ & $439.6 \pm 14.5^{* * *}$ & $347.5 \pm 8.7$ & $431.6 \pm 25.7^{* * *}$ & $391.6 \pm 13.7$ & $467.4 \pm 15.6^{* * *}$ \\
\hline Intra-abdominal fat pad weights (g) & $29.2 \pm 4.4$ & $39.3 \pm 3.7^{* *}$ & $26.6 \pm 2.6$ & $40.2 \pm 4.9^{* *}$ & $38.7 \pm 3.8$ & $43.8 \pm 2.6^{* *}$ \\
\hline Food intake (kcal/day) & $82.7 \pm 4.3$ & $99.8 \pm 4.2^{* * *}$ & $81 \pm 2.5$ & $95.7 \pm 7.0^{* * *}$ & $94.9 \pm 4.7^{\dagger \delta}$ & $110.1 \pm 4.2^{* * *+\delta}$ \\
\hline Uterus (g) & $0.50 \pm 0.03$ & $0.10 \pm 0.01^{* * *}$ & $0.51 \pm 0.07$ & $0.10 \pm 0.01^{* * *}$ & $0.47 \pm 0.05$ & $0.11 \pm 0.01^{* * *}$ \\
\hline
\end{tabular}

SD standard diet, HF + Chol (0.25\%) high fat + cholesterol, Ovx ovariectomised, Sham sham operated

Values are mean \pm SE with $n=8$ rats per group

${ }^{* *}$ Significantly different from the sham groups $(P<0.01)$, ${ }^{* * *}(P<0.001)$

${ }^{\dagger}$ Significantly different from SD diet $(P<0.05)$

${ }^{\delta}$ Significantly different from SD+Chol diet $(P<0.05)$ 
(a)

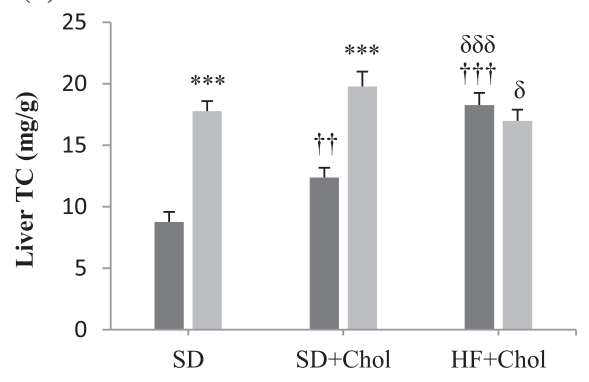

(c)

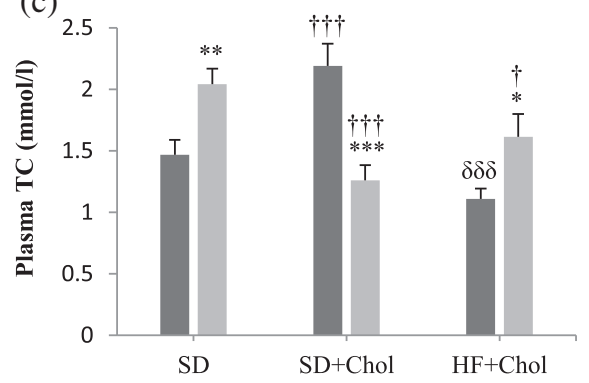

(b)

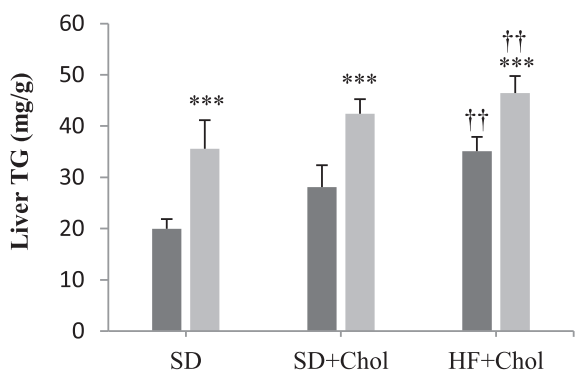

(d)

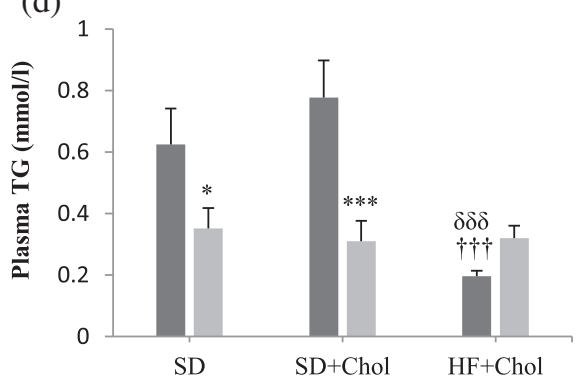

Fig 1 Liver and plasma total cholesterol (TC) and triacylglycerol (TG) levels in sham (-) and ovariectomized (Ovx, gray square symbol) rats fed a standard diet (SD), a SD $+0.25 \%$ cholesterol diet $(S D+C h o l)$, and a high fat $+0.25 \%$ cholesterol diet $(H F+C h o l)$. Values are mean \pm SE with $n=8$ rats per group. * Significantly different from respective Sham rats $(P<0.05),{ }^{* *}(P<0.01),{ }^{* * *}(P<0.001) ;{ }^{\dagger}$ Significantly different from respective rats fed the SD diet $(P<0.05),{ }^{+\dagger}(P<0.01),{ }^{\dagger+}(P<0.001)$; ${ }^{\delta}$ Significantly different from respective rats fed the $\mathrm{SD}+\mathrm{Chol}$ diet $(P<0.05),{ }^{\delta \delta \delta}(P<0.001)$

found no difference between Ovx and Sham rats in any of the measured gene expression in all dietary conditions (Fig. 3). However, we observed that several of the genes (Abcg8, Mdr2, Bsep, Fxr, and Ntcp) had their transcripts decreased when animals were fed the SD + Chol compared to the SD diet. On the other hand, Abcg5/g8 and Cyp7a1 mRNA levels were higher $(P<0.01)$ in $\mathrm{HF}+$ Chol compared to SD + Chol fed animals.

To further explore hepatic cholesterol metabolism, we measured gene transcripts of key molecules involved in cholesterol uptake from cholesterol rich lipoproteins. We first found a lower $(P<0.001)$ Lrp1 gene expression in Ovx than in Sham animals in all dietary groups (Fig. 4(b)). Interestingly, we observed that gene expression of Ldlr and Srebp2 were highly decreased $(P<$ 0.001 ) when rats were fed either the $\mathrm{SD}+\mathrm{Chol}$ or the $\mathrm{HF}+\mathrm{Chol}$ diets as compared to rats fed the SD di et, while $\operatorname{Lrp} 1$ transcripts were decreased $(P<0.05)$ only under the SD + Chol diet (Fig. 4).

\section{Discussion}

The main finding of the present study is that high dietary cholesterol represses gene expression of key molecular markers of VLDL synthesis (Mttp, Acat2, Apob-100) in Sham rats and even more so in Ovx rats (Mttp and Apob-100). In addition, the sole addition of cholesterol to the SD diet reduced gene expression of several liver markers of bile acid and phospholipid transport (Bsep, Ntcp, Mdr2) in both Sham and Ovx animals. Finally, gene expression of key markers involved in liver LDL cholesterol uptake ( $L d l-r$ and $L r p 1$ ) was also decreased by the sole addition of cholesterol to the diet in Sham as well as in Ovx rats. These results first indicate that the cholesterol component in a mixed diet is a determinant factor that regulates liver cholesterol metabolism in sham as well as in Ovx rats. In addition, the present molecular responses to high cholesterol diet converge to indicate a reduction in hepatic TG and cholesterol excretion from the liver, this being to a certain extent, accentuated by the absence of estrogens.

We recently reported data showing an impairment of VLDL assembly following ovariectomy and high fat/high cholesterol diets in rats [15]. The present study extends these findings by indicating that the sole high content of cholesterol in the diet impaired VLDL assembly in Sham and even more so in Ovx animals. Ovx has been previously associated with a decrease in VLDL production in rat via Mttp regulation [20]. Accordingly, molecular expression of several genes related to VLDL assembly, including Mttp and Apob, was reduced in Ovx rats under the present SD diet. That high cholesterol diet decreases even more so VLDL assembly/production in Ovx animals suggests an additive effect of these two stimuli. This additive effect on VLDL assembly/production is 

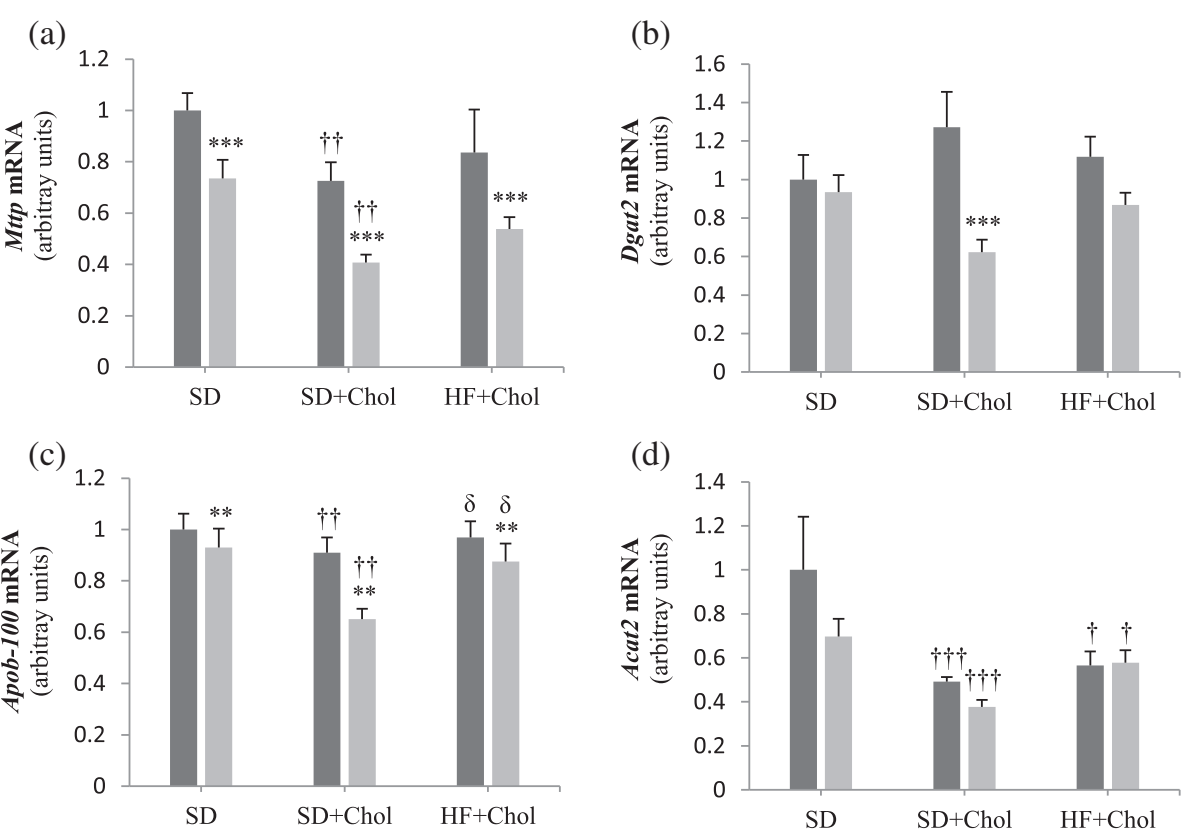

(e)

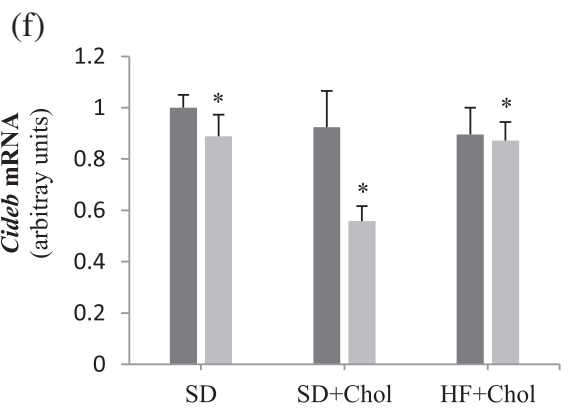

Fig 2 Hepatic mRNA expression of genes involved in VLDL synthesis/production in sham ( $\mathbf{m})$ and ovariectomized (Ovx, gray square symbol) rats fed a standard diet (SD), a SD + $0.25 \%$ cholesterol diet (SD + Chol), and a high fat $+0.25 \%$ cholesterol diet (HF + Chol). Values are mean \pm SE with $n=8$ rats per group. ${ }^{* *}$ Significantly different from respective Sham rats $(P<0.001)$; ${ }^{\dagger}$ Significantly different from rats fed the respective SD diet $(P<0.05){ }^{++}(P<0.01),{ }^{++\dagger}(P<0.001) ;{ }^{\delta \delta}$ Significantly different from rats fed the respective SD + Chol diet $(P<0.01)$. Mttp microsomal TG transfer protein; Dgat-2, diacylglycerol acyl transferase-2; apoB-100, apolipoprotein B-100; Acat-2, acyl-coA cholesterol acyl transferase-2; Sar1a, small GTP-binding protein; Cideb, cell death-inducing like-effector type B

corroborated by the higher accumulation of TC and TG in liver along with lower levels of plasma TC and TG found in Ovx compared to Sham animals under the $\mathrm{SD}+$ Chol diet.

It is not clear at this point the mechanism by which a high cholesterol diet would repress VLDL assembly/ production. Hepatic VLDL production has been reported to be reduced by competitive inhibitors of HMG-CoA reductase, the main enzyme responsible for cholesterol biosynthesis, through the regulation of the SREBP family transcription factors [21, 22]. SREBP-2 gene expression was repressed by the cholesterol diet in the present study. Although there is evidence that several steps of the VLDL assembly/secretion are under the control of SREBP-1, there are also indications that upon the cell type and physiological conditions,
SREBP1 and SREBP2 may mediate changes in lipoprotein assembly and secretion [22]. An alternative explanation for the decrease in VLDL synthesis/production following high dietary cholesterol and Ovx would be the endoplasmic reticulum (ER) stress. There are indications that cholesterol can induce hepatic ER stress through free cholesterol accumulation in the ER [23], and that ER stress limits VLDL assembly and secretion through apoB degradation [24].

In addition of reducing VLDL synthesis/production, high cholesterol feeding in Ovx and Sham animals also repressed gene expression of key markers of bile acid metabolism. The present finding of a reduction in Bsep and $M d r 2$ gene expression suggests a reduction in bile acid and phospholipid excretion, while the reduction in Ntcp mRNA suggests a reduction in bile acid uptake from the entero-hepatic circulation. These observations 

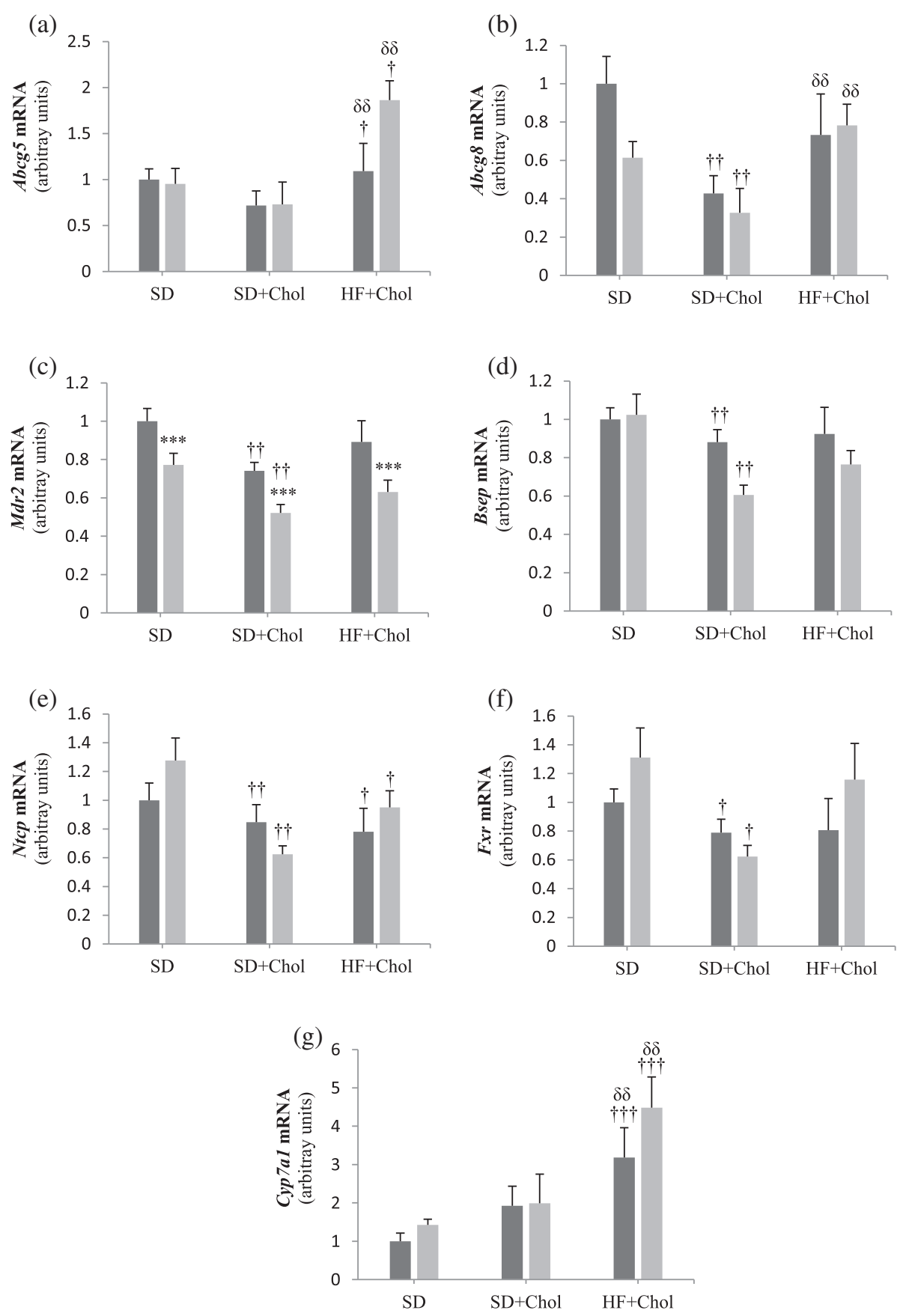

Fig. 3 Hepatic mRNA expression of genes related to bile acid metabolism in sham ( $\mathbf{a})$ and ovariectomized (Ovx, gray square symbol) rats fed a standard diet (SD), a SD $+0.25 \%$ cholesterol diet (SD + Chol), and a high fat $+0.25 \%$ cholesterol diet (HF + Chol). Values are mean \pm SE with $n=8$ rats per group. ${ }^{* *}$ Significantly different from respective Sham rats $(P<0.001)$; ${ }^{\dagger}$ Significantly different from rats fed the respective SD diet $(P<0.05),{ }^{\dagger+}(P<0.01),{ }^{\dagger+}(P<0.001) ;{ }^{\delta \delta}$ Significantly different from rats fed the respective SD + Chol diet $(P<0.01),{ }^{\delta \delta \delta}(P<0.001) . A b c g 5 / A b c g 8$, ATP-cassette binding protein G5 and G8; Mdr2, multidrug resistance-associated transporter 2; Bsep, bile salt export pump; Ntcp, Na + -taurocholate cotransporting polypeptide; Cyp7a1, cytochrome P450 7A1; Fxr, farnesoid X receptor

may be taken as an indication of a reduction of the entero-hepatic circulation of bile acids. Furthermore, the reduction in Fxr gene expression in the SD + Chol fed rats suggests that even though cholesterol level was increased in liver, there was no accumulation of bile acids since the role of hepatic Fxr is to prevent bile acid hepatotoxicity. Dietary interventions such as high cholesterol/high fat diets have been reported to repress Fxr gene expression in liver $[13,15]$. The gene expression of the present key molecules thus supports the previous 


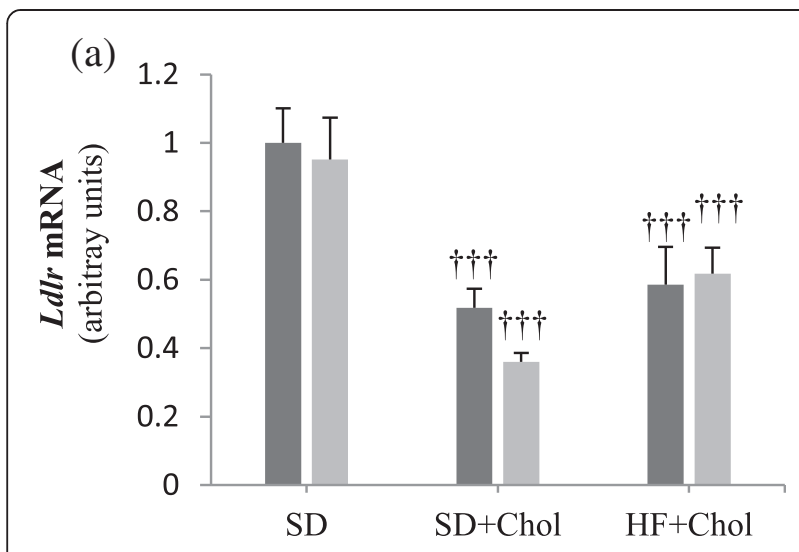

(b)
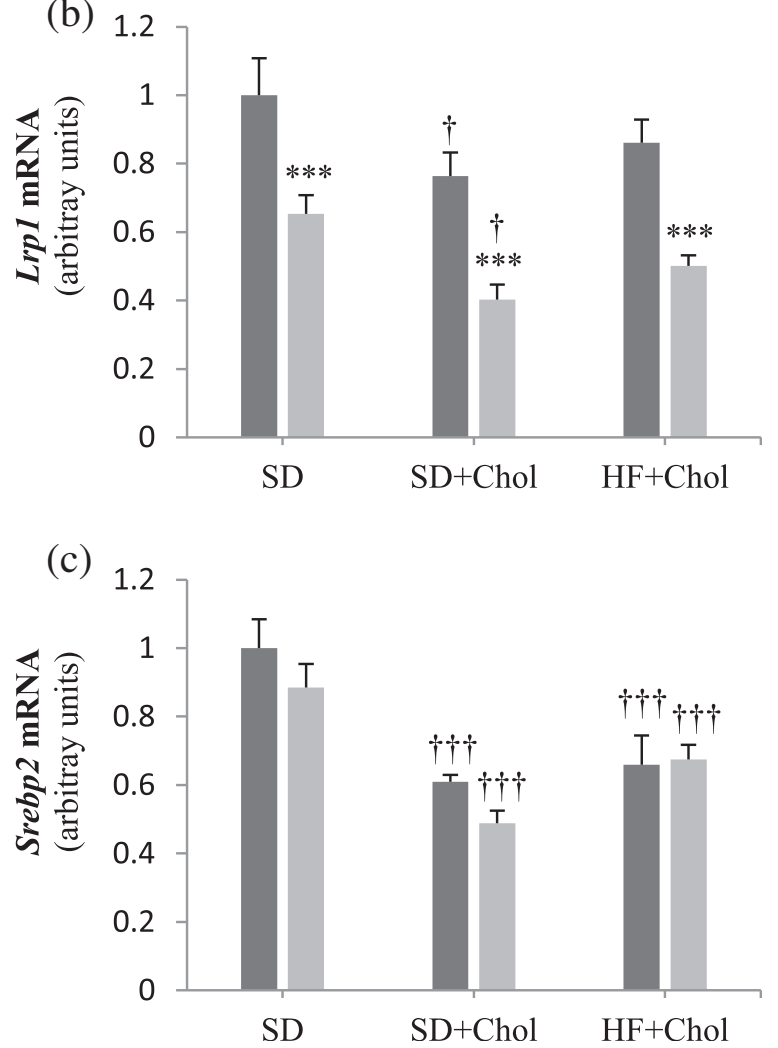

Fig. 4 Hepatic mRNA expression of genes involved in uptake of cholesterol rich lipoproteins from the circulation in sham (a) and ovariectomized (Ovx, gray square symbol) rats fed a standard diet (SD), a SD + $0.25 \%$ cholesterol diet (SD + Chol), and a high fat $+0.25 \%$ cholesterol diet $(H F+C h o l)$. Values are mean \pm SE with $n=8$ rats per group. ***Significantly different from respective Sham rats $(P<0.001)$; ${ }^{+}$Significantly different from rats fed the respective SD diet $(P<0.05)$, ${ }^{++\dagger}(P<0.001)$. Ldlr, LDL-receptor; Lrp-1, LDL receptor-related protein-1; Srebp2, sterol regulatory element-binding protein-2

suggestion that high cholesterol feeding in rats disrupts bile acid metabolism [15].

In addition to a decrease in gene expression of markers of VLDL synthesis and bile acid transport, dietary cholesterol in the present study resulted in a down regulation in gene expression of $L d l-r$ and $L r p 1$ in Sham and Ovx animals suggesting a decrease in cholesterol uptake from circulation. This response was even more pronounced in Ovx rats for Lrp1 transcripts. Ovx has been previously reported to be associated with a reduction in the expression of several genes involved in the uptake of lipoprotein molecules [11, 25]. The decrease in LDL receptors was most likely linked to the excess hepatic cholesterol level through the decrease in Srebp2 gene expression [26, 27]. Taken together, the present results suggest an association between reduced VLDL synthesis/production, reduced bile acids transport and reduced LDL receptors under high dietary cholesterol in Sham as well as in Ovx rat.

The interest of comparing a high cholesterol diet with and without the addition of a high fat content is enlighten by the observation that the sole addition of cholesterol to a SD diet had no effect on body weight in Sham and Ovx animals while the addition of fat in the diet caused an increase in food intake and a strong tendency in higher body weight $(P=0.06)$ and intra-abdominal $(P=0.08)$ fat accumulation (Table 3). This implies, on a clinical point of view, that a high cholesterol diet might not be perceived as being deleterious since it does not affect body weight when in fact it causes several metabolic perturbations. One noticeable effect of adding fat to cholesterol in the diet was the increase in liver fat accumulation resulting from the diet and most likely from increased lipogenesis [28]. On the other hand, higher expression of $A b c g 5 / g 8$ and Cyp $7 a 1$ were also observed under $\mathrm{HF}+\mathrm{Chol}$ feeding, both of these genes being involved in cholesterol excretion from the liver. These responses may be taken as an indication that hepatic cholesterol metabolism may be less vulnerable to high fat/high cholesterol feeding than high dietary cholesterol alone.

The present results have limitations that need to be taken into consideration. First, the limited duration (5 weeks) of the present experimental conditions might represent an acute situation that may lead to different results on a longer term basis with a chronic adaptation to the diet. The present results generated from an animal model are intended to open research issues that need to be tested in humans.

In summary, results of the present study first indicate that gene expressions of key markers of VLDL synthesis/ production are reduced under high cholesterol feeding and that this reduction is exacerbated in Ovx animals. In addition, the present data provide evidence that the activities of bile acid and $L d l-r$ pathways are also reduced by the sole addition of cholesterol to a SD diet in Sham as well as in Ovx animals. These results point to the direction as if the liver under high cholesterol feeding reduces its excretion of cholesterol, at least on a short term basis, thus contributing to exacerbate liver fat and cholesterol accumulation known to occur in Ovx animals. 


\section{Competing interests}

The authors declare that they have no competing interests.

\section{Authors' contributions}

This work was performed at the University of Montreal. ZF (conception, design, acquisition of data), IC and ETNS. (acquisition of data) JML (conception, design). All authors contributed to data interpretation and drafting/revising the article for important intellectual content. All authors approved the final version to be published.

\section{Financial support}

This work was supported by grants from the Natural Sciences and Engineering Research Council of Canada (NSERC; 7594).

Received: 22 August 2015 Accepted: 3 October 2015

\section{Published online: 09 October 2015}

\section{References}

1. Volzke H, Schwarz S, Baumeister SE, Wallaschofski H, Schwahn C, Grabe HJ, et al. Menopausal status and hepatic steatosis in a general female population. Gut. 2007:56:594-5.

2. Paquette A, Shinoda M, Rabasa Lhoret R, Prud'homme D, Lavoie JM. Time course of liver lipid infiltration in ovariectomized rats: impact of a high-fat diet. Maturitas. 2007:58:182-90.

3. Picard F, Deshaies Y, Lalonde J, Samson P, Labrie C, Bélanger A, et al. Effects of the estrogen antagonist EM-652. $\mathrm{HCl}$ on energy balance and lipid metabolism in ovariectomized rats. Int J Obes Relat Metab Disord. 2000;24:830-40.

4. Deshaies Y, Dagnault A, Lalonde J, Richard D. Interaction of corticosterone and gonadal steroids on lipid deposition in the female rat. Am J Physiol. 1997;273:E355-362.

5. Kimura T, Matsumoto T, Akiyoshi M, Owa Y, Miyasaka N, Aso T, et al. Body fat and blood lipids in postmenopausal women are related to resting autonomic nervous system activity. Eur J Appl Physiol. 2006;97:542-7.

6. Kaur A, Jindal S, Kaur IP, Chopra K. Effect of sesamol on the pathophysiological changes induced by surgical menopause in rodents. Climacteric. 2013;16(4):426-37.

7. Chaudhuri A, Borade NG, Hazra SK. A study of heart rate variablity tests and lipid profile in postmenopausal women. J Indian Med Assoc. 2012;110(4):230-2.

8. Park Y, Kwon HY, Shimi MK, Rhyu MR, Lee Y. Improved lipid profile in ovariectomized rats by red ginseng extract. Pharmazie. 2011;66(6):450-3.

9. Kato M, Ogawa H, Kishida T, Ebihara K. The mechanism of the cholesterollowering effect of water-insoluble fish protein in ovariectomised rats. Br J Nutr. 2009;102:816-24.

10. Kamada Y, Kiso S, Yoshida Y, Chatani N, Kizu T, Hamano M, et al. Estrogen deficiency worsens steatohepatitis in mice fed high-fat and high-cholesterol diet. Am J Physiol Gastrointest Liver Physiol. 2011;301:G1031-1043.

11. Ngo Sock ET, Chapados NA, Lavoie J-M. LDL receptor and Pcsk9 transcripts are decreased in liver of ovariectomized rats: Effects of exercise training. Horm Metab Res. 2014;46(8):550-5.

12. Côté I, Ngo Sock ET, Lévy É, Lavoie J-M. An atherogenic diet decreases liver FXR gene expression and causes severe hepatic steatosis and hepatic cholesterol accumulation: effect of endurance training. Eur J Nutr. 2013;52(5):1523-32.

13. Savard C, Tartaglione EV, Kuver R, Haigh WG, Farrell GC, Subramanian S, et al. Synergistic interaction of dietary cholesterol and dietary fat in inducing experimental steatohepatitis. Hepatology. 2013;57(1):81-92.

14. Ngo Sock ET, Cote I, Mentor JS, Prud'homme D, Bergeron R, Lavoie J-M. Ovariectomy stimulates hepatic fat and cholesterol accumulation in high-fat diet-fed rats. Horm Metab Res. 2013;45(4):283-90.

15. Côté I, Chapados NA, Lavoie J-M. Impaired VLDL assembly: a novel mechanism contributing to hepatic lipid accumulation following ovariectomy and high-fat/high-cholesterol diets. Br J Nutr. 2014;112(10):1592-600.

16. Ouguerram $K$, Chetiveaux M, Zair $Y$, Costet $P$, Abifadel M, Varret $M$, et al. Apolipoprotein B100 metabolism in autosomal-dominant hypercholesterolemia related to mutations in PCSK9. Arterioscler Thromb Vasc Biol. 2004;24(8):1448-53.
17. Rohlmann A, Goothardt M, Hammer RE, Herz J. Inducible inactivation of hepatic LRP gene by cre-mediated recombination confirms role of LRP in clearance of chylomicrons remnants. J Clin Invest. 1998;101(3):689-95.

18. Robertson MC, Owens RE, Klindt J, Friesen HG. Ovariectomy leads to a rapid increase in rat placental lactogen secretion. Endocrinology. 1984;114(5):1805-11.

19. Folch J, Lees M, Sloane Stanley GH. A simple method for the isolation and purification of total lipids from animal tissues. J Biol Chem. 1957;226(1):497-509.

20. Barsalani R, Chapados NA, Lavoie J-M. Hepatic VLDL-TG production and MTP gene expression are decreased in ovariectomized rats: effects of exercise training. Horm Metab Res. 2010;42:860-7.

21. Kang S, Davis RA. Cholesterol and hepatic lipoprotein assembly and secretion. Biochim Biophys Acta. 2000;1529:223-30

22. Wang S-L, Du E, Martin TD, Davis RA. Coordinate regulation of lipogenesis, the assembly and secretion of apolipoprotein B-containing lipoproteins by sterol response element binding protein 1. J Biol Chem. 1997;272(31):19351-8.

23. Hager L, Li L, Pun H, Liu L, Hossain MA, Maguire GF, et al. Lecithin:cholesterol acyltransferase deficiency protects against cholesterol-induced hepatic endoplasmic reticulum stress in mice. J Biol Chem. 2012;287(24):20755-68.

24. Ota T, Gayet C, Ginsberg HN. Inhibition of apolipoprotein B 100 secretion by lipid-induced hepatic endoplasmic reticulum stress in rodents. J Clin Invest. 2008;118:316-32

25. Ge XZ, Tian PF, Lin Q, Huo Q. The influence of soybean isoflavone on expression of low density lipoprotein receptor (LDLR) mRNA in ovariectomized rats. Zhong Yao Cai. 2006; 29: 349-351.

26. Dubac C, Chamberland A, Wassef H, Davignon J, Seidah NG, Bernier L, et al. Statins upregulate PCSK9, the gene encoding the proprotein convertase neural apoptosis-regulated convertase-1 implicated in familial hypercholesterolemia. Arterioscler Thromb Vasc Biol. 2004;24(8):1454-9.

27. Smith JR, Osborne TF, Goldstein JL, Brown MS. Identification of nucleotides responsible for enhancer activity of sterol regulatory element in low density lipoprotein receptor gene. J Biol Chem. 1990;265(4):2306-10.

28. Repa JJ, Liang G, Ou J, Bashmakov Y, Lobaccaro JM, Shimomura I, et al. Regulation of mouse sterol regulatory element-binding protein-1c gene (SREBP-1c) by oxysterols receptors, LXRalpha and LXRbeta. Gene Dev. 2000;14(22):2819-30.

\section{Submit your next manuscript to BioMed Central and take full advantage of:}

- Convenient online submission

- Thorough peer review

- No space constraints or color figure charges

- Immediate publication on acceptance

- Inclusion in PubMed, CAS, Scopus and Google Scholar

- Research which is freely available for redistribution 